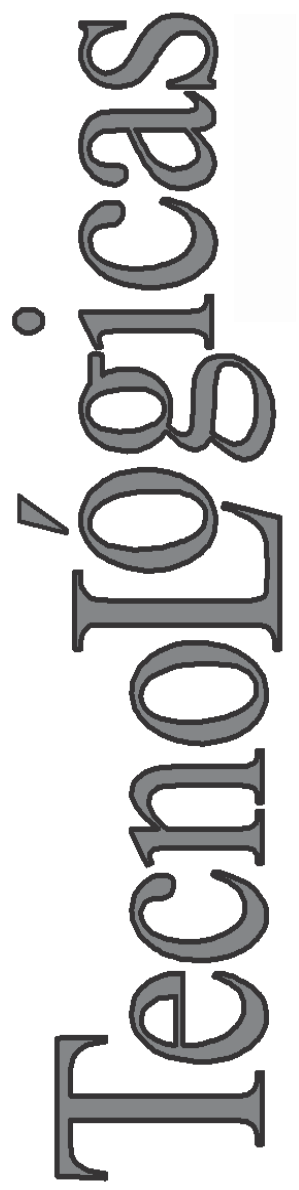

\title{
Compensador de Iluminación Utilizando un Diodo LED como Sensor y Actuador de Luz
}

\section{Lighting Compensator Using a Diode LED as Light Sensor and Actuator}

Roger A. Martínez-Ciro ${ }^{1}$ Alejandro Restrepo-Martínez ${ }^{2}$ Francisco E. Lopez-Giraldo ${ }^{3}$

1 Facultad de Ingeniería, Instituto Tecnológico Metropolitano, Medellín-Colombia alexanders-rogers@hotmail.com

2 Grupo de Investigación en Automática, Electrónica y

Ciencias Computacionales, Instituto Tecnológico Metropolitano, Medellín-Colombia alejandromartinez@itm.edu.co

3 Grupo de Investigación en Automática, Electrónica y

Ciencias Computacionales, Instituto Tecnológico

Metropolitano, Medellín-Colombia franciscolopez@itm.edu.co 


\title{
Resumen
}

En este trabajo se presenta un sistema electrónico capaz de controlar la polarización directa e inversa aplicada en las terminales de un Diodo Emisor de Luz (LED) lo cual permite configurarlo como actuador y sensor de luz conmutadamente, con la finalidad de diseñar un sistema que permita compensar niveles de potencia lumínica a un valor fijado por el usuario. Se describe el diseño electrónico conformado por un bloque análogo y un procesador de bajo desempeño Arduino UNO, que permitió validar: el funcionamiento del LED en su dualidad de operación y el algoritmo implementado. Para el montaje desarrollado fue posible definir una ecuación experimental que permite determinar el nivel de potencia lumínica que debe ser aportado por el LED compensador, adicionalmente se calculó el porcentaje PWM que debe ser aplicado en sus terminales. A través del montaje implementado fue posible hacer la medición de la fotocorriente entregada por el LED configurado como sensor de luz y posteriormente convertirla a voltaje por medio del acondicionamiento análogo. Finalmente se logró un algoritmo que permite realizar la compensación de potencia lumínica.

\section{Palabras clave}

LED sensor; LED actuador; polarización directa; polarización inversa; fotocorriente.

\begin{abstract}
This work presents an electronic system capable of controlling the direct and inverse polarization applied to the terminals of a LED diode; this allows configuring it as an actuator and light sensor interchangeably. The final goal is to design a system that compensates levels, set by the user, of luminesce potency. The design is described by an Analog block and a low-tech processor Arduino UNO. The Arduino UNO allowed for the functioning of the LED to operate dually by the implemented algorithm. An equation for the developed set was defined experimentally which determines the luminesce potency that the compensating LED should provide; the duty cycle for the PWM applied to the LED terminals was calculated too. Through the developed set it was possible to measure the photocurrent given by the LED configured as light sensor and then turn it into a voltage through the analog block. FInally an algorithm was obtained to compensate luminesce potency.
\end{abstract}

\section{Keywords}

LED sensor; LED actuator; polarization direct; inverse polarization; photocurrent. 


\section{INTRODUCCIÓN}

Debido al gran desarrollo tecnológico en torno a los Diodos Emisores de Luz (Light Emitting Diode - LED), estos se han convertido en dispositivos electrónicos muy utilizados en cuanto a indicadores luminosos y en sistemas de iluminación, debido a que son de poco consumo de corriente eléctrica, compactos, eficientes y económicos. En efecto, ha despertado el interés en algunos investigadores, de estudiar el comportamiento espectral de los LEDs utilizando diferentes técnicas para la caracterización de la emisión de luz (Morocho et al., 2012). Una de las aplicaciones más comunes de los LEDs se encuentra en el desarrollo de sistemas de iluminación, los cuales están remplazado las bombillas tradicionales de alto consumo energético, más específicamente las lámparas alógenas; esto ha permitido disminuir un $63 \%$ el consumo energético (Alonso et al., 2012). Por otra parte, y en busca de dar un uso más eficiente a la energía eléctrica, se han propuesto sistemas de iluminación regulados utilizando LEDs, y diferentes técnicas para el control de potencia lumínica (Matta \& Mahmud, 2010; Milashevski et al., 2012). Los trabajos descritos, indican que los LEDs han sido utilizados convencionalmente como fuentes lumínicas, ya que fueron diseñados especialmente para suplir esta tarea; sin embargo, hace más de 30 años se han venido empleando como sensores de luz espectralmente selectivos, con la finalidad de solucionar problemas en el campo de la fotometría solar (Mims, 2009; Brooks et al 2007).

En ninguna de las referencias mencionadas anteriormente se ha configurado el LED como Sensor y Actuador de luz Conmutadamente (LED-SAC), ya que es muy común su utilización como actuador de luz en sistemas de iluminación, ó, como sensor espectralmente selectivo.

En el presente trabajo se realiza este tipo de configuración para construir un sistema de iluminación compensado, dicho sistema implica diseñar e implementar un hardware electrónico que permita caracterizar y validar el funcionamiento del diodo LED en su dualidad de operación. La importancia de utilizar un LED-SAC, radica en la posibilidad de diseñar lámparas autoreguladas para el ahorro de energía eléctrica, donde los mismos LEDs podrán ser 
utilizados como sensores de luz con el fin de determinar la intensidad lumínica del medio.

\section{MONTAJE EXPERIMENTAL}

El montaje experimental consiste en dos LEDs Azules con longitud de onda pico de 440.370nm y un ancho de banda de $12.59 \mathrm{~nm}$, dicha longitud de onda fue medida con el espectrómetro LR1-600-3 de Aseq Instruments®. El LED Compensador (LED-C) es configurado en su dualidad de funcionamiento LED-SAC por medio del bloque electrónico; paralelamente, dicho bloque electrónico configura el LED Actuador como fuente de luz, aplicando en sus terminales una señal PWM (Pulse Width Modulation) para regular la potencia lumínica de emisión. Posteriormente se incorporó un medidor de potencia lumínica PM100DThorlabs ${ }^{\circledR}$ en el centro de la caja, con el cual fue posible monitorear la emisión de luz de los LEDs de manera conjunta e independiente. Dicho montaje se muestra en la Fig. 1.

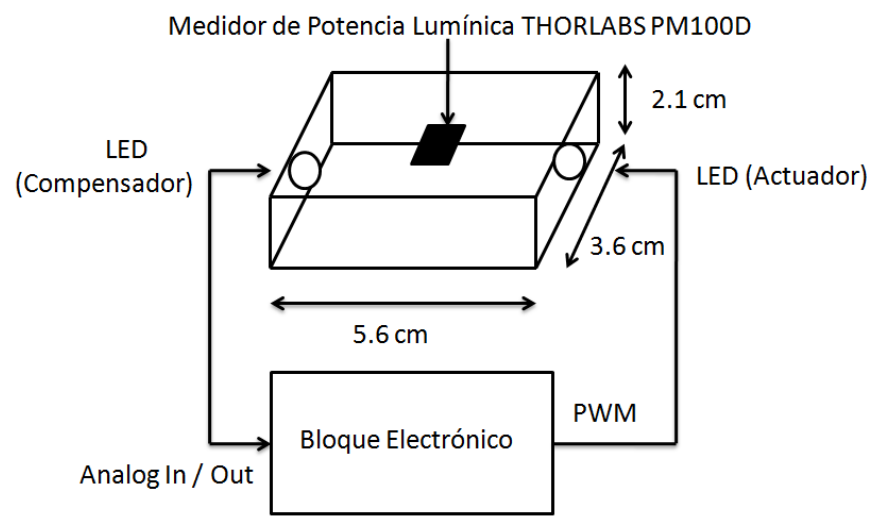

Fig. 1. Montaje experimental del Compensador de Iluminación. Fuente: Autores 


\subsection{Bloque Electrónico}

Para llevar a cabo el compensador de iluminación se requiere de un conjunto de subsistemas electrónicos. A continuación se describe cada uno: el primero está conformado por un aislador electrónico, el cual tiene como función, controlar la polaridad directa e inversa del LED-C y convertirlo en actuador o sensor de luz respectivamente, como se ilustra en la Fig. 2.

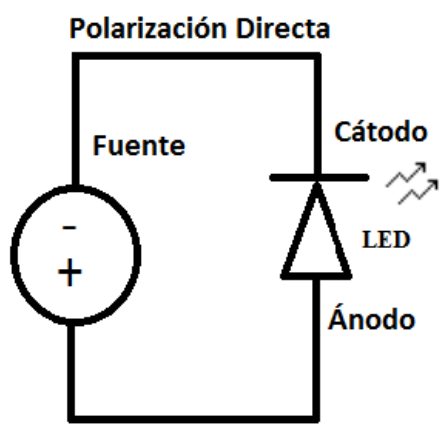

LED Actuador de Luz

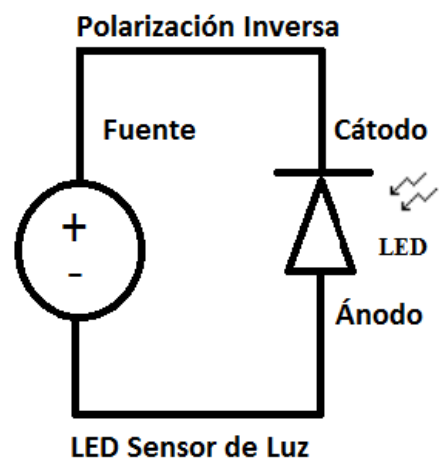

Fig. 2. Polarización directa e inversa del diodo LED. Fuente: Autores

Luego de realizar dicho procedimiento y configurar el LED como sensor, se utiliza el amplificador transimpedance para convertir la fotocorriente proveniente del sensor en voltaje y al mismo tiempo realizar el acople de impedancias; el tercer subsistema es el amplificador de señal, el cual tiene como objetivo aumentar los niveles de voltaje obtenidos anteriormente, y los configura en el rango de trabajo del conversor $\mathrm{A} / \mathrm{D}(4.8 \mathrm{mv}$ y $5 \mathrm{v})$; el conversor $\mathrm{A} / \mathrm{D}$ recibe la señal análoga acondicionada y la digitaliza a una resolución de 10 bits (1023 valores por muestra); dichos datos son almacenados en la memoria del microcontrolador del ARDUINO UNO, y transmitidos por puerto serial al computador; finalmente se construyen las ecuaciones que facilitan el cálculo del porcentaje PWM que debe ser aplicado al LED-C. El procedimiento descrito se muestra en la Fig. 3. 


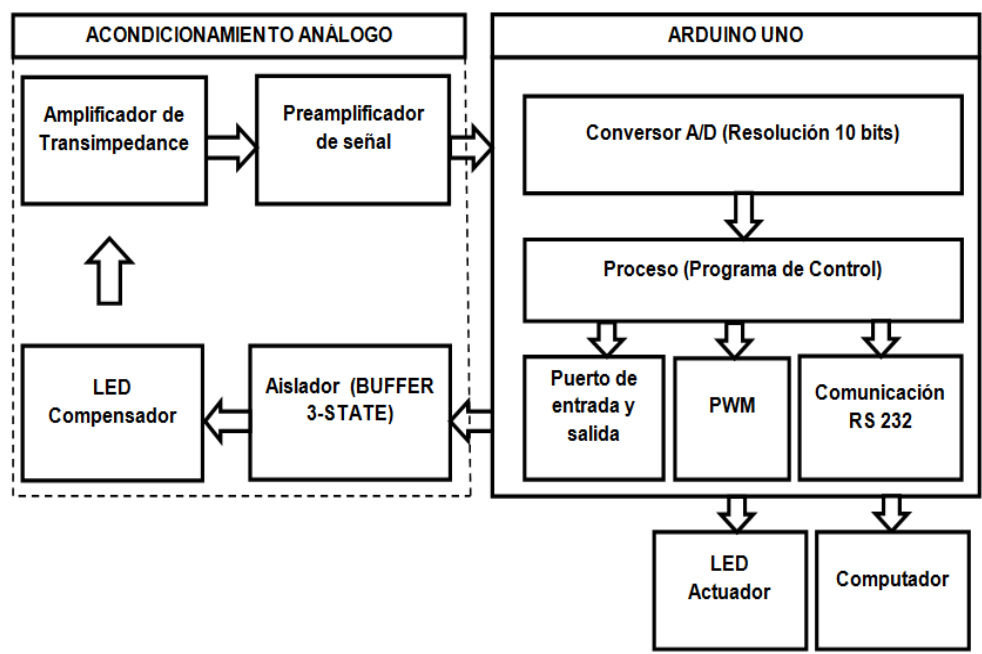

Fig. 3. Sistema de Acondicionamiento Electrónico. Fuente: Autores

\section{ALGORITMO DEL COMPENSADOR DE LUZ}

El compensador de luz se llevó a cabo desde diferentes etapas: Primero se realiza la calibración del LED-C configurado como sensor de luz y se registra en un vector de datos el voltaje máximo y mínimo producido por este, dicho voltaje está en función de la potencia lumínica emitida por el LED actuador; luego se procede a caracterizar ambos LEDs (actuador y compensador), para ello se configuran como emisores de luz y se registra la potencia lumínica de cada uno; la última etapa consiste en relacionar los datos almacenados anteriormente, con la finalidad de construir una ecuación experimental que permitirá calcular el porcentaje PWM que debe ser aplicado al LED-C para realizar el ajuste lumínico.

\subsection{Resultado y Discusión}

Para desarrollar el compensador de luz, se parte del hecho de que el sistema cuenta con dos LEDs (LED compensador y LED actuador) donde el primero es configurado en su dualidad de funcionamiento (sensor - actuador) y el restante es utilizado única- 
mente como fuente de luz, los instrumentos de medida y dispositivos electrónicos fueron explicados en el numeral anterior. Antes de comenzar los experimentos de medición de potencia y detección con el LED-C configurado como sensor de luz, se realizó la medición de la respuesta espectral y la longitud de onda en la cual está trabajando dicho sistema. Para ello se midió el espectro de emisión de luz del diodo LED utilizando el espectrómetro LR1-600-3 de Aseq Instruments ${ }^{\circledR}$, como se puede apreciar en Fig. 4.

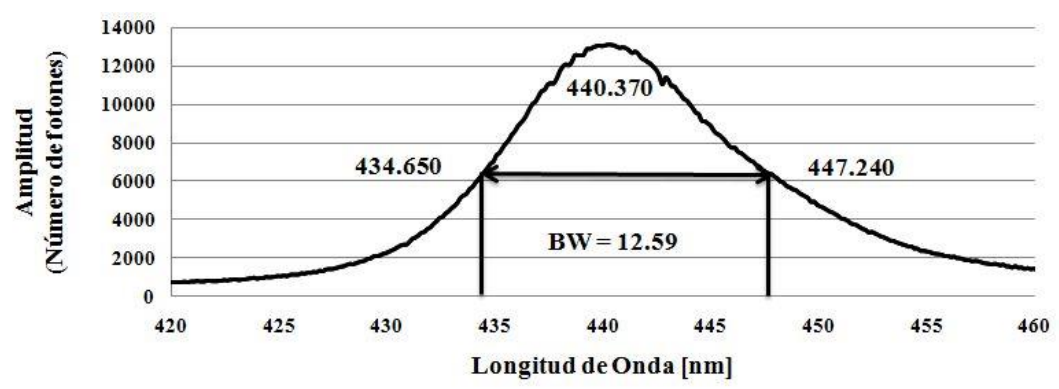

Fig. 4. Respuesta espectral del diodo LED azul. Fuente: Autores

Para determinar el ancho de banda (Band Width - BW) de emisión del LED, se tiene en cuenta lo siguiente: longitud de onda pico (440.370nm), en este punto se mide la amplitud (A) y se convierte en $\mathrm{dB}$, luego se le resta $3 \mathrm{~dB}$ que es el equivalente a la mitad de la señal como se indica en (1); posteriormente se fijan los valores correspondientes a la longitud de onda de cada lateral, con los cuales se calcula el BW (12.59dB) realizando la diferencia entre la longitud de onda superior (LOS) $(447.240 \mathrm{~nm})$ y la longitud de onda inferior (LOI) $(434.650 \mathrm{~nm})$ como se indica en (2).

$A(d B)=10 \log (A)-3 d B$

$\mathrm{BW}=\mathrm{LOS}-\mathrm{LOI}$

Conociendo el comportamiento espectral del LED en términos de longitud de onda, se procede a la caracterización del sistema. Dicha caracterización consiste en realizar la medición del voltaje entregado por el LED-C al configurarlo como sensor de luz, y la 
medición de potencia lumínica al configurar los dos LEDs como actuadores de luz.

En la Fig. 5 se muestra la relación que existe entre la potencia lumínica emitida por el LED actuador (en adelante se denominará PotAct) con respecto al voltaje inducido en el sensor (en adelante se denominará VolSens), posteriormente se aplicó la línea de tendencia a dicha gráfica y se extrajo la ecuación que establece la relación entre estas dos variables, como se muestra en (3). El error cuadrático (0.9999) indica que la respuesta voltaica del sensor, aumenta a un ritmo constante con respecto al incremento de la potencia lumínica del LED actuador.

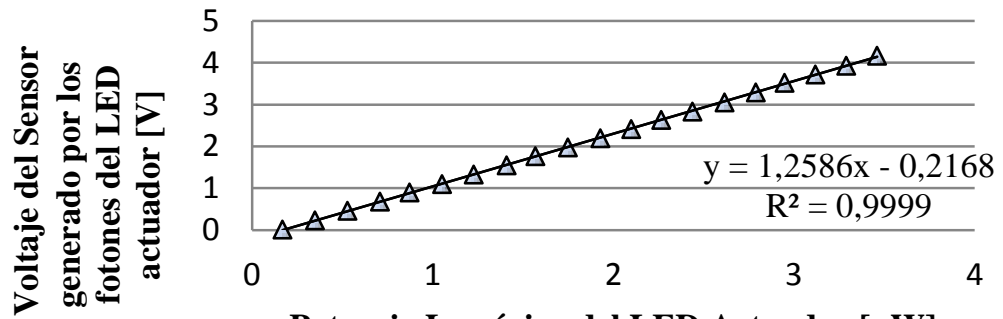

Potencia Lumínica del LED Actuador $[\mu \mathrm{W}]$

Fig. 5. Respuesta voltaica del LED compensador configurado como sensor de luz midiendo la emisión del LED actuador. Fuente: Autores

PotAct $=\frac{\text { VolSens }+0.2168}{1.2586}$

En la Fig. 6 se presenta la medición de potencia lumínica emitida por cada LED y la sumatoria de potencia cuando ambos emiten al mismo tiempo y al mismo porcentaje PWM; posteriormente se aplica la regresión lineal a los datos del LED-C y se extrae la ecuación que permite calcular el porcentaje PWM (\%PWM) con respecto a la potencia lumínica de emisión del LED compensador (PotComp), como se muestra en (4).

$\% \mathrm{PWM}=\frac{\text { PotComp }-0.0263}{0.0919}$ 


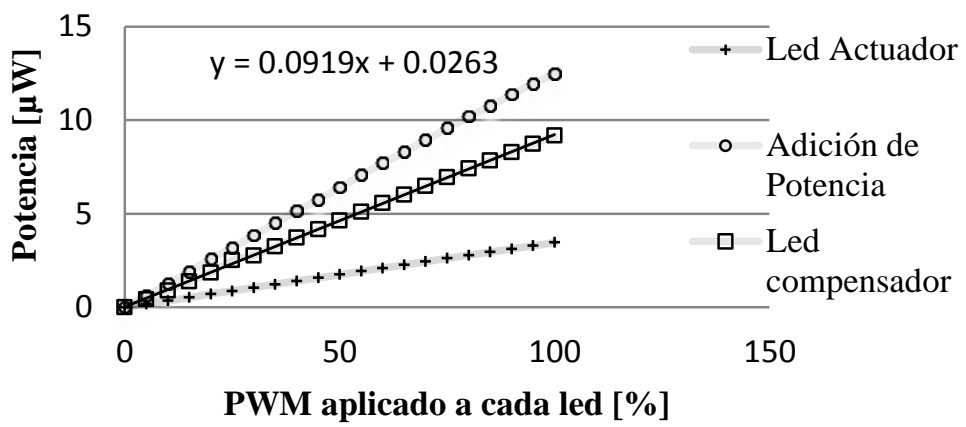

Fig. 6. Relación entre la potencia lumínica aportada por cada LED y la sumatoria. Fuente: Autores

\subsection{Validación de Ecuaciones y el Funcionamiento del Compensador de Luz}

El primer paso consiste en definir el nivel de referencia del sistema en términos de potencia lumínica, el cual se denomina (NvelR). Luego se procede a determinar la potencia lumínica a la cual se encuentra emitiendo el LED actuador (PotAct); como dicha potencia no es controlada por el sistema, para ello se procede a configurar el LED-C como sensor de luz y se realiza la lectura del voltaje entregado por éste (Volsens), finalmente se calcula la potencia utilizando (3), mostrada anteriormente. El siguiente paso es determinar la potencia lumínica que debe aportar el LED-C al configurarlo como actuador de luz para llegar al nivel de referencia establecido por el usuario, esto se realiza utilizando (5). Finalmente se calcular el porcentaje PWM (\%PWM) que debe ser aplicado en las terminales del LED-C al configurarlo como actuador de luz, teniendo en cuenta la potencia lumínica (PotComp) que debe aportar al sistema, para ello se utiliza (4) mostrada anteriormente.

PotComp $=$ NvelR - PotAct

Para verificar la ecuación experimental se leen los datos almacenados en el sistema y se realiza el cálculo del porcentaje PWM, los cuales son comparados con los porcentajes de PWM almacenados en los vectores. Esta comparación se realizó de manera gráfica, obteniéndose los resultados mostrados en la Fig. 7. 


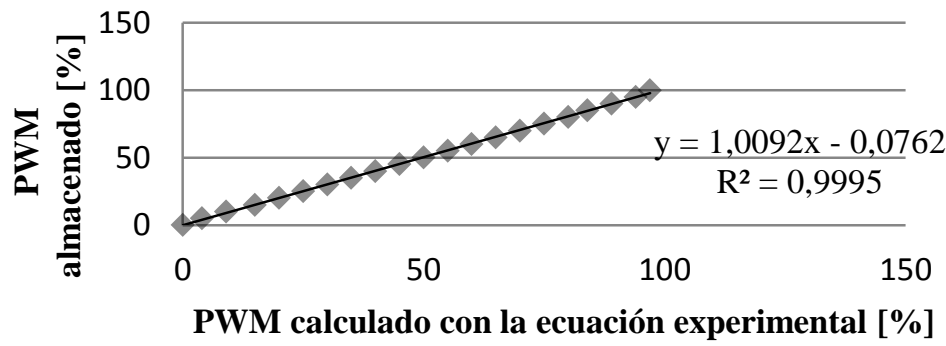

Fig. 7. Comparación entre el porcentaje PWM calculado con la ecuación vs porcentaje PWM almacenado en el sistema. Fuente: Autores

Los valores calculados deberían ser iguales a los datos almacenados, lo cual indica que la pendiente debería ser igual a 1 . Dicha pendiente no es exactamente igual a 1 ya que existe un error del $0.05 \%$ en los datos calculados experimentalmente con respecto a los datos almacenados, esto se debe a que la ecuación se utilizó sin realizar ningún tipo de ajuste o calibración que permitiera disminuir dicho error. Sin embargo, dicho porcentaje de error no genera mayores efectos en las emisiones lumínicas del LED compensador. Con lo cual se dice que el sistema planteado es confiable.

\section{CONCLUSIONES}

El sistema electrónico implementado permitió utilizar la dualidad del diodo LED para compensar niveles de luz, tal como se demostró en los cálculos del porcentaje PWM utilizando la ecuación experimental, los cuales fueron comparados con los almacenados en los vectores del sistema.

Los valores calculados del porcentaje PWM difieren en un $0.05 \%$ con respecto a los datos almacenados en el sistema: esto se debe a que las ecuaciones experimentales planteadas no poseen un nivel de ajuste para corregir dicho error. Adicionalmente dichos datos se encuentran en punto flotante, los cuales deben ser redondeados a la unidad más próxima para calcular el porcentaje PWM, el cual debe ser un número entero y positivo.

Los resultados de porcentaje PWM calculados con las ecuaciones indican que si es posible realizar una compensación de niveles 
lumínicos, teniendo caracterizado el sistema en términos de la potencia lumínica existente en el medio y la fotocorriente que esta induce en el LED sensor.

\section{REFERENCIAS}

Alonso, J.M., Gacio, D., Calleja, A.J., Ribas, J., Corominas, E., (2012); A Study on LED Retrofit Solutions for Low-Voltage Halogen Cycle Lamps, IEEE TRANSACTIONS ON INDUSTRY APPLICATIONS, (48), No. 5.

Morocho, M.A., Hernández, C., (2012); Caracterización Óptica de Diodos emisores de Luz mediante su espectro de emisión y patrones de radiación, Scientia et Technica, XVII (51). ISSN 0122-1701.

Milashevski, I., Tetervenok, O., Suzdalenko, A., (2012); Comparative Study of LED Ballasts for Different Light Regularion Techniques, 15th International Power Electronics and Motion Control Conference, EPE-PEMC 2012 ECCE Europe, Novi Sad, Serbia.

Matta, S., Mahmud, S.M., (2010); An Intelligent Light Control System for Power Saving, IECON 2011 - 37th Annual Conference on IEEE Industrial Electronics Society, On page(s): 3316 - 3321.

Mims, F.M., (2009); LED Sun Photometry, Optics and Photonics News, (20), No. 9, 32-38.

Brooks, R. David., Mims, F.M., Roettger, Richard., (2007); Inexpensive Near-IR Sun Photometer for Measuring Total Column Water Vapor, Journal of Atmospheric and Oceanic Technology, Vol. 24, page(s): 1268-1276. 